\title{
Estimation of the pressure at a listener's ears in an active headrest system using the remote microphone technique
}

\author{
Woomin Jung, ${ }^{*}$ Stephen J. Elliott, and Jordan Cheer \\ Institute of Sound and Vibration Research, \\ University of Southampton, SO17 1BJ, United Kingdom
}

(Dated: March 27, 2018)

\begin{abstract}
The remote microphone technique is considered in this paper as a way of estimating the error signals at a listener's ears in an active headrest system, using remotely installed monitoring microphones. A least-squares formulation for the optimal observation filter is presented, including a regularization factor that is chosen to satisfy both the estimation accuracy and robustness to uncertainties. The accuracy of the nearfield estimation is first investigated for a diffuse field via simulations. Additionally, simulations of a free field are also used to investigate the effect of the spatial directivity of the primary field. Finally, experiments in an anechoic chamber are conducted with 24 monitoring microphones around a dummy head positioned in an active headrest system. When six loudspeakers, driven by uncorrelated random disturbances, are used to generate the primary field, the best arrangement of monitoring microphones is considered, taking into account both accuracy and robustness.
\end{abstract}

PACS numbers: PACS: 43.38.-p, 43.50.Ki, 43.60.Rw

\footnotetext{
*wj3e13@soton.ac.uk; Corresponding author.
} 


\section{INTRODUCTION}

The objective of local active sound control in enclosures is generally to reduce the acoustic potential energy at particular locations, which produces localized zones of quiet around controlled error sensors. Previous research on active headrest systems, as a practical configuration of local control, has shown that this system potentially extends the controllable frequency range and the attenuation performance at desired positions, such as at the ears of a listener, compared to global active sound control [1] [2] [3] [4]. The advantages of a local active headrest system, over a global active control system, may be listed as [2] [5]:

- The secondary loudspeakers and error sensors are acoustically well-coupled and so the loudspeakers do not have to drive too hard to control the error signals. As a result, noise enhancement at other positions can be low.

- The proximity between the secondary loudspeakers and error sensors can reduce the acoustic propagation delay in the control path, so that is shorter than the propagation time associated with the primary disturbance signals and this allows active control process to remain causal despite electrical delays in the control system.

- The plant response, which is the acoustic transfer response between the secondary loudspeakers and error sensors, is relatively simple and largely determined by the local geometry since the direct field of the secondary source is dominant over the reverberant field. Consequently, ill-conditioning can be avoided and the control system can be more robust to small perturbations in the response of the enclosure.

Despite these advantages, the direct installation of error sensors in the desired positions has limited the practical application of the active headrest system. For example, passengers in a car are unlikely to accept the need to wear in-ear microphones over long periods of time for local active control. In addition, the zone of quiet around a fixed error sensor, within which more than $10 \mathrm{~dB}$ attenuation is achieved, is limited to a diameter of about $1 / 10$ of an acoustic wavelength in a diffuse field, which is about $3.4 \mathrm{~cm}$ at $1 \mathrm{kHz}[6]$. Therefore, any separation between the error sensors and the targeted control regions can lead to the zone of quiet being outside of the desired region of control, and noise enhancement may even be generated due to the constructive interference between the disturbance and control signals. A wide variety of virtual sensing algorithms have been proposed to overcome this problem, by estimating the pressures that would be measured at microphones located in the control- 
targeted regions, which are referred to as virtual error sensors, from remotely installed physical sensors, which are referred to as monitoring sensors. The virtual microphone arrangement, [3] [7] [8], assumes that the disturbance signals at the virtual error sensors are almost equal to those at the monitoring sensors, although the plant responses from the secondary sources to the monitoring and virtual sensors are significantly different due to the nearfield of the secondary sources. With this assumption, Garcia-Bonito et al. [8] have shown, in the context of the attenuation performance of an active headrest system in a diffuse sound field, that at frequencies, below about $500 \mathrm{~Hz}$, the attenuation performance achieved using the virtual microphone arrangement is comparable to that achieved when error signals at the ears of a dummy head are used directly, since the primary acoustic fields at the virtual and monitoring sensors are similar. At frequencies from $500 \mathrm{~Hz}$ to $1 \mathrm{kHz}$, however, the substantial difference between the primary acoustic fields at the virtual and monitoring sensors limits the attenuation performance with the virtual microphone arrangement.

The remote microphone technique was initially suggested by Roure and Albarrazin [9] and has also been widely investigated in the literature [4] [10] [11]. To overcome the limitation of the virtual microphone arrangement at higher frequencies, the remote microphone technique applies a specific filter, called the observation filter here, to estimate the disturbance signals at the virtual error sensors by optimally weighting the disturbance signals at the monitoring sensors. In a preliminary identification stage, physical error sensors are typically installed at the positions of the virtual error sensors and the disturbance signals are simultaneously measured at both the error sensors and monitoring sensors. From these signals, the observation filter can be designed in either the frequency or time domain [10] [11] [12]. After this initial training, the physical error sensors can be removed and the disturbance signals at the virtual error sensors are estimated by filtering the disturbance signals at the monitoring sensors with the observation filter. The attenuation performance of an active headrest system using either the virtual microphone arrangement or the remote microphone technique has been compared by Das et al. [4] who showed that about $20 \mathrm{~dB}$ more reduction is achieved with the remote microphone technique, compared with the virtual microphone arrangement, with a tonal primary noise of frequency of around $196 \mathrm{~Hz}$ and a feedback active headrest system. Although a relatively low frequency tonal noise was controlled in this study, it was clear that a more accurate estimation of the virtual error signals, using the remote microphone technique, can significantly improve the attenuation performance because the 
feedback control system is particularly affected by inaccurate error signals. The accuracy of the remote microphone technique depends on the spatial properties of the primary acoustic field, however, and this needs to be investigated to determine the attenuation performance and the convergence and stability of the active control system in different applications.

Other virtual sensing algorithms have been suggested, including the forward difference extrapolation technique [13] [14], and the Kalman filtering virtual sensing method [15] [16]. In the forward difference extrapolation technique, pressure and pressure gradient at the virtual error sensor are estimated by fitting a spatial polynomial to the signals measured at a number of monitoring sensors, but the estimation accuracy is limited to low frequencies, for which the distance between the virtual and monitoring sensors is smaller than the wavelength. In the Kalman filtering virtual sensing method, the active control responses are modelled using a state-space system and estimates of the plant states are calculated using measured error signals at the monitoring sensors to estimate signals at the virtual error sensors. These alternative methods were compared with the remote microphone technique in $[10]$.

This paper considers the remote microphone technique, with an observation filter calculated using the least-squares method, when different arrays of monitoring microphones are used in different sound fields, since the accuracy of the nearfield estimation is mainly determined by the spatial properties of the primary acoustic field. It is shown that an appropriate regularization factor is necessary to achieve nearfield estimation that is robust to both acoustic and experimental uncertainties and this can be obtained from the trade-off between the accuracy of the nearfield estimation and the robustness to uncertainties. An understanding of the performance of the remote microphone technique in different environments is important, since this is one of the main limitation on the alternative achieved using local active control without physical error microphones.

In Section II, the optimal observation filter for the remote microphone technique is formulated in the frequency domain. The disturbances are assumed to be random and stationary and can thus be described by their power and cross spectral densities. If the disturbances are broadband random, the causality of an observation filter needs to be considered and the corresponding formulation can be obtained in the time domain as a matrix of finite impulse response filters, as suggested by [11] [17]. However, if the disturbance signals have a narrowband spectrum, the frequency domain formulation can be applied without the causality 


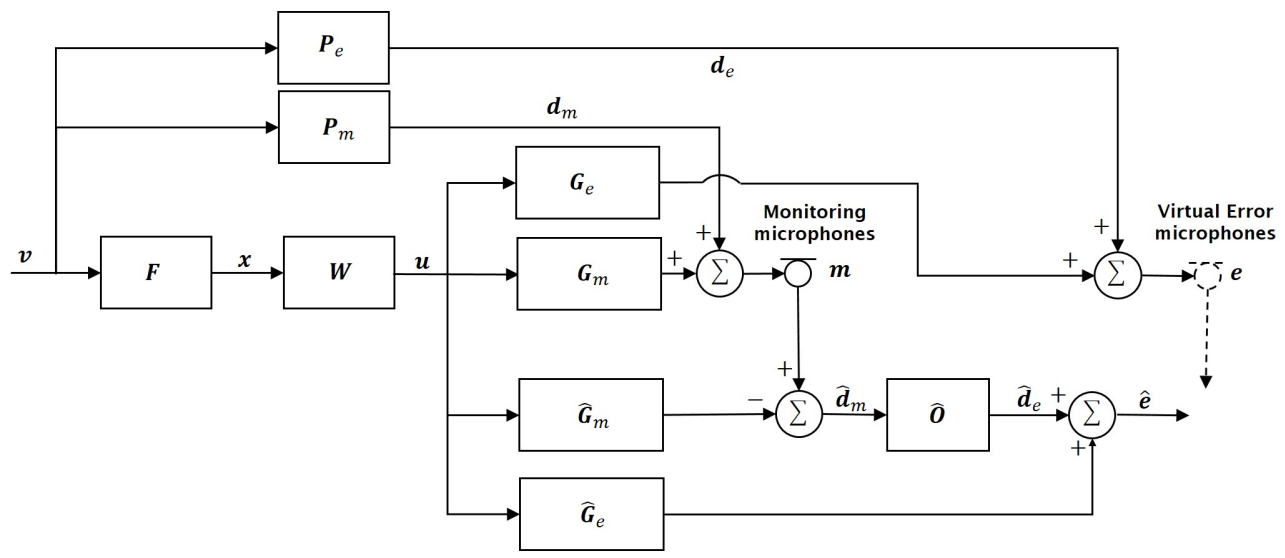

FIG. 1. Block diagram of a feedforward control system using the remote microphone technique. a o 99 O

\section{Se} 2 technique in an anechoic chamber. Finally, Section V presents the conclusions of this work.

\section{THE REMOTE MICROPHONE TECHNIQUE}

The remote microphone technique, which estimates virtual error signals from the signals measured at the remote monitoring microphones, can be combined with a feedforward active control system, as shown in Fig. 1 . The $N_{v}$ primary sources, which for random disturbances have a vector of complex source strengths in a single frequency bin of $\mathbf{v}=\left[\begin{array}{llll}v_{1}, v_{2} & \ldots & v_{N_{v}}\end{array}\right]^{\mathrm{T}}$,

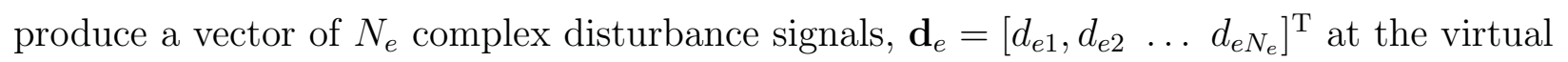
error microphones and a vector of $N_{x}$ reference signals, $\mathbf{x}=\left[x_{1}, x_{2} \ldots x_{N_{x}}\right]^{\mathrm{T}}$ at the reference microphones via the matrices of transfer responses $\mathbf{P}_{e}$ and $\mathbf{R}$, respectively. The reference signals drive the matrix of control filters, $\mathbf{W}$, to produce a vector of $N_{u}$ control signals, $\mathbf{u}=\left[\begin{array}{lll}u_{1}, u_{2} & \ldots & u_{N_{u}}\end{array}\right]^{\mathrm{T}}$, which are transmitted to the virtual error microphones, via the matrix of complex plant responses, $\mathbf{G}_{e}$, to minimize these disturbance signals. The vector of complex error signals, e, at the virtual error microphones after control can thus be defined as 


$$
\mathbf{e}=\mathbf{d}_{e}+\mathbf{G}_{e} \mathbf{u}=\mathbf{d}_{e}+\mathbf{G}_{e} \mathbf{W} \mathbf{x}=\mathbf{P}_{e} \mathbf{v}+\mathbf{G}_{e} \mathbf{W R v}
$$

where the dependence on frequency of all of these signals and responses has been suppressed for notational convenience. Any feedback from the secondary sources to the reference sensors is assumed to be negligible, which can be ensured through the use of feedback cancellation filters for example. When the direct measurement of the virtual error signals, e, is infeasible, they can be estimated from the $N_{m}$ complex monitoring microphone signals, $\mathbf{m}=\left[\begin{array}{llll}m_{1}, m_{2} & \ldots & m_{N_{m}}\end{array}\right]^{\mathrm{T}}$, which are equal to

$$
\mathbf{m}=\mathbf{d}_{m}+\mathbf{G}_{m} \mathbf{u}=\mathbf{d}_{m}+\mathbf{G}_{m} \mathbf{W} \mathbf{x}=\mathbf{P}_{m} \mathbf{v}+\mathbf{G}_{m} \mathbf{W R v}
$$

where $\mathbf{d}_{m}=\left[\begin{array}{llll}d_{m 1}, & d_{m 2} & \ldots & d_{m N_{m}}\end{array}\right]^{\mathrm{T}}$ is a vector of disturbance signals measured at the monitoring microphones and $\mathbf{G}_{m}$ is the matrix of plant responses between the secondary sources and the monitoring microphones. In practice, true matrices of plant responses, $\mathbf{G}_{e}$ and $\mathbf{G}_{m}$ are not usually available and therefore estimates of the plant responses are used in the controller, as denoted by $\hat{\mathbf{G}}_{e}$ and $\hat{\mathbf{G}}_{m}$. Similarly $\mathbf{d}_{m}$ and $\mathbf{d}_{e}$ are measured in practice as $\hat{\mathbf{d}}_{m}$ and $\hat{\mathbf{d}}_{e}$. If $\hat{\mathbf{O}}$ is the estimated observation filter used to calculate $\hat{\mathbf{d}}_{e}$ from $\hat{\mathbf{d}}_{m}$, the estimated error signals, $\hat{\mathbf{e}}$, at the virtual error microphones can be written as

$$
\hat{\mathbf{e}}=\hat{\mathbf{d}}_{e}+\hat{\mathbf{G}}_{e} \mathbf{u}=\hat{\mathbf{O}} \hat{\mathbf{d}}_{m}+\hat{\mathbf{G}}_{e} \mathbf{u}=\hat{\mathbf{O}}\left(\mathbf{m}-\hat{\mathbf{G}}_{m} \mathbf{u}\right)+\hat{\mathbf{G}}_{e} \mathbf{u},
$$

where in all cases the superscript ^ represents an estimate of an actual value.

The observation filter in Eq. (3) will influence how accurately $\hat{\mathbf{d}}_{e}$ is estimated, and the estimation error between $\hat{\mathbf{d}}_{e}$ and $\mathbf{d}_{e}$ is an important factor in determining the performance of the control system. The optimal observation filter, $\mathbf{O}_{\text {opt }}$, can be derived by minimizing the mean squared estimation error between $\mathbf{d}_{e}$ and $\hat{\mathbf{d}}_{e}$. In practice, however, it is also necessary to include a term in the cost function that is proportional to the mean squared magnitude of all the coefficients in the observation filter, to improve the robustness of this filter. If $\mathbf{d}_{e}$ and $\mathbf{d}_{m}$ are measured in preliminary experiments before active control, so that in this case $\mathbf{d}_{m}$ is known, $\mathbf{O}_{\text {opt }}$ can be obtained by minimizing the cost function [11], 


$$
\begin{gathered}
J_{1}=\operatorname{trace}\left\{E\left[\left(\mathbf{d}_{e}-\mathbf{O d}_{m}\right)\left(\mathbf{d}_{e}-\mathbf{O} \mathbf{d}_{m}\right)^{\mathrm{H}}+\beta \mathbf{O O}^{\mathrm{H}}\right]\right\} \\
=\operatorname{trace}\left\{\mathbf{S}_{d_{e} d_{e}}-\mathbf{S}_{d_{m} d_{e}} \mathbf{O}^{\mathrm{H}}-\mathbf{O S}_{d_{m} d_{e}}^{\mathrm{H}}+\mathbf{O}\left(\mathbf{S}_{d_{m} d_{m}}+\beta \mathbf{I}\right) \mathbf{O}^{\mathrm{H}}\right\},
\end{gathered}
$$

where $E[]$ is the expectation operator, ${ }^{\mathrm{H}}$ is the Hermitian, complex conjugate, transpose and $\beta$ is a positive real regularizing parameter, $\mathbf{I}$ is the identity matrix, $\mathbf{S}_{d_{e} d_{e}}=E\left[\mathbf{d}_{e} \mathbf{d}_{e}^{\mathrm{H}}\right]$ and $\mathbf{S}_{d_{m} d_{m}}=E\left[\mathbf{d}_{m} \mathbf{d}_{m}^{\mathrm{H}}\right]$ are the power spectral density matrices for $\mathbf{d}_{e}$ and $\mathbf{d}_{m}$ respectively, and $\mathbf{S}_{d_{m} d_{e}}=E\left[\mathbf{d}_{e} \mathbf{d}_{m}^{\mathrm{H}}\right]$ is the cross spectral density matrix between $\mathbf{d}_{m}$ and $\mathbf{d}_{e}$. In a stationary random sound field, when the number of monitoring microphones $\left(N_{m}\right)$ is larger than the number of error microphones $\left(N_{e}\right)$, the problem of minimizing Eq. (4) is mathematically overdetermined. The term, $\beta \mathbf{O O}^{\mathrm{H}}$, in Eq. (4a) always ensures that the matrix, $\mathbf{S}_{d_{m} d_{m}}+\beta \mathbf{I}$, in Eq. (4b) is positive definite, and hence invertable. As a result, the cost function $J_{1}$, which is a quadratic function of the real and imaginary parts of each element in $\mathbf{O}$, has a unique minimum value. The optimal value, $\mathbf{O}_{\mathrm{opt}}$, can be calculated using similar methods to those described in Ref. [18] to give

$$
\mathbf{O}_{\mathrm{opt}}=\mathbf{S}_{d_{m} d_{e}}\left(\mathbf{S}_{d_{m} d_{m}}+\beta \mathbf{I}\right)^{-1}=\mathbf{P}_{e} \mathbf{S}_{v v} \mathbf{P}_{m}^{\mathrm{H}}\left(\mathbf{P}_{m} \mathbf{S}_{v v} \mathbf{P}_{m}^{\mathrm{H}}+\beta \mathbf{I}\right)^{-1},
$$

where $\mathbf{S}_{v v}$ is the power spectral density matrix of the primary source strengths, $\mathbf{v}$, and $\mathbf{P}_{e}$ and $\mathbf{P}_{m}$ are the matrices of acoustical transfer responses from the primary sources to the error microphones and monitoring microphones respectively, as shown in Fig. 1. When practical estimates of $\mathbf{P}_{e}$ and $\mathbf{P}_{m}$ are used to calculate the observation filter in Eq. (5b), this is denoted $\hat{\mathbf{O}}_{\text {opt }}$, as used in Eq. (3).

Although the term, $\mathbf{S}_{d_{m} d_{m}}+\beta \mathbf{I}$ in Eq. (5a) is positive definite, its inverse can be illconditioned, so that $\mathrm{O}_{\mathrm{opt}}$ is sensitive to both physical and numerical uncertainties. A reduction in the condition number can be obtained by the appropriate selection of the regularization factor, $\beta$. As $\beta$ increases, the solution for the optimal observation filter becomes more robust to practical uncertainties [19], but an excessively large regularization factor can produce a biased solution, with a higher estimation error. Therefore, to select an appropriate regularization factor, it is necessary to consider the trade-off between the robustness and the accuracy with which $\hat{\mathbf{d}}_{e}$ estimates $\mathbf{d}_{e}$. 
The normalized mean squared estimation error level at the virtual microphone, $L_{\epsilon}$, can be defined as

$$
L_{\epsilon}=10 \log _{10}\left|\frac{S_{\epsilon \epsilon}}{S_{d_{e} d_{e}}}\right|,
$$

where $\epsilon$ is defined as $d_{e}-\hat{d}_{e}$ at a single virtual microphone location.

\section{NUMERICAL SIMULATIONS IN A DIFFUSE FIELD}

\section{A. Estimating the disturbance at a single virtual error microphone with a single} monitoring microphone

When the complex disturbance signal, $d_{m}\left(\mathbf{x}_{1}\right)$, at a single monitoring microphone at a point described by the coordinate vector, $\mathbf{x}_{1}$, is used to estimate the complex disturbance signal, $d_{e}\left(\mathbf{x}_{2}\right)$, at a single error microphone at a point, $\mathbf{x}_{2}$, the accuracy depends on the spatial cross-correlation function between the two signals, which for a diffuse primary field, as derived in [2] [20], can be written as

$$
<d_{e}\left(\mathbf{x}_{2}\right) d_{m}^{*}\left(\mathbf{x}_{1}\right)>=<|p|^{2}>[\sin (k \Delta x)] /(k \Delta x)=<|p|^{2}>\operatorname{sinc} k \Delta x
$$

where $<>$ denotes the operation of spatial averaging by taking samples of the pressure field over a volume of space with dimensions much larger than an acoustic wavelength, * indicates complex conjugation, $\left\langle|p|^{2}>\right.$ is the space-averaged mean squared pressure in the diffuse field, $k$ is the wavenumber and $\Delta x$ is the Euclidean distance between $\mathbf{x}_{1}$ and $\mathbf{x}_{2}$.

If only a single monitoring microphone is used, $\mathbf{S}_{d_{m} d_{m}}$ is a positive scalar and thus Eq. (5) can be used with $\beta$ set to zero, with Eq. (7), to give the space-averaged optimal observation filter, $\mathrm{O}_{\text {opt }}(\Delta x)$ in a diffuse field as

$$
\mathrm{O}_{\text {opt }}(\Delta x)=<d_{e}\left(\mathbf{x}_{2}\right) d_{m}^{*}\left(\mathbf{x}_{1}\right)><d_{m}\left(\mathbf{x}_{1}\right) d_{m}^{*}\left(\mathbf{x}_{1}\right)>^{-1}=\operatorname{sinc} k \Delta x
$$

Using Eq. (8) for the nearfield estimation, the space-averaged estimation error between $d_{e}\left(\mathbf{x}_{2}\right)$ and $\hat{d}_{e}\left(\mathbf{x}_{2}\right)$ is given by 
(a)

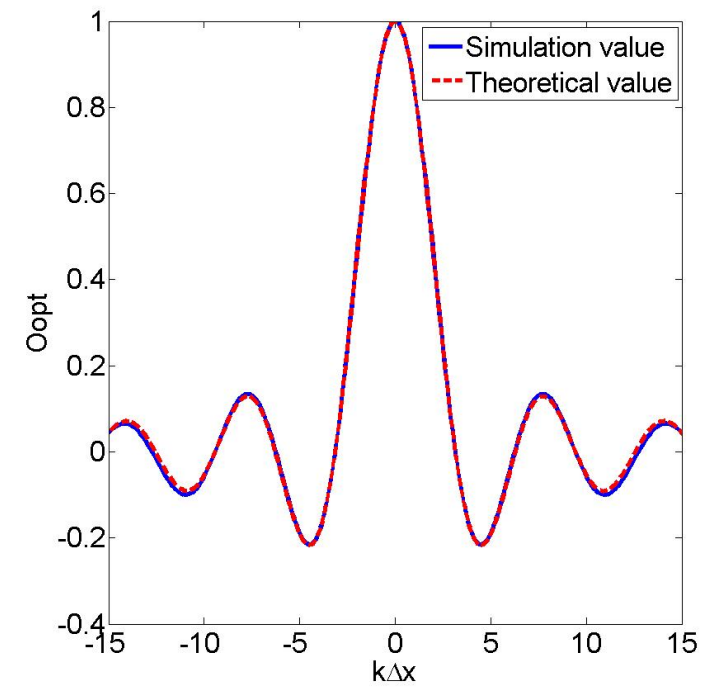

(b)

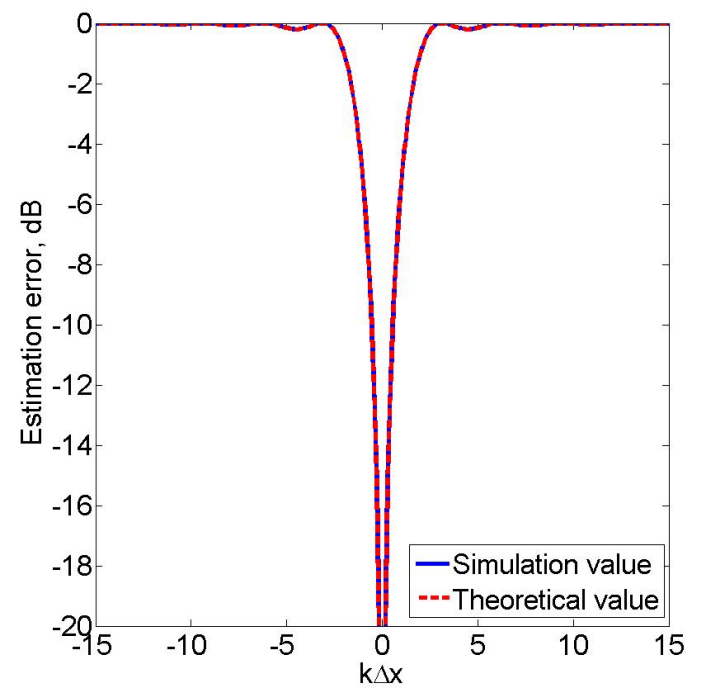

FIG. 2. (a) The response of the observation filter and (b) the estimation error when a single monitoring microphone is used to estimate the disturbance signals at a single error sensor in a diffuse field. Simulation (solid line), Theoretical value (dashed line)

$$
\begin{aligned}
L_{\epsilon} & =10 \log _{10}\left(\frac{<\left|d_{e}\left(\mathbf{x}_{2}\right)-\mathrm{O}_{\mathrm{opt}}(\Delta x) d_{m}\left(\mathbf{x}_{1}\right)\right|^{2}>}{<\left|d_{e}\left(\mathbf{x}_{2}\right)\right|^{2}>}\right) \\
& =10 \log _{10}\left(1-\operatorname{sinc}^{2} k \Delta x\right) .
\end{aligned}
$$

To confirm these predictions, a series of simulations have been implemented in a synthetic diffuse field, as in [11], using 642 uncorrelated monopole sources uniformly distributed over a sphere of radius $3 \mathrm{~m}$ around the monitoring and error microphones. The observation filter and estimation error are calculated from the disturbance signals of a single monitoring microphone at the center of the sphere and a $51 \times 51$ grid of potential virtual error microphone locations, in the $1 \mathrm{~m} \times 1 \mathrm{~m}$ region. The results of this simulation are presented in Fig. 2 and show that there is a good agreement between the analytical and simulation results. The estimation error is significantly greater than $-10 \mathrm{~dB}$ as $k \Delta x$ is increased beyond about \pm 0.55 , so that $\Delta x$ is greater than about $1 / 11.4$ of a wavelength, which occurs when either the frequency is increased or the distance between the monitoring and virtual error microphones is increased. 


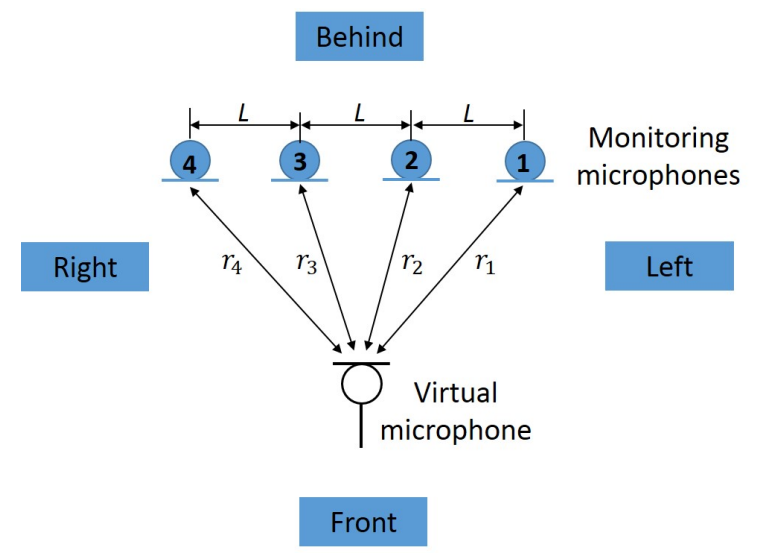

FIG. 3. Geometry of a linear array with four monitoring microphones used to estimate the pressure at a given virtual microphone location.

B. Estimating the disturbance at a single virtual error microphone using multiple monitoring microphones

To demonstrate the performance with multiple monitoring microphones, simulations were performed using four monitoring microphones to estimate the signal at a single virtual error microphone in a diffuse field. Assuming that there are four monitoring microphones arranged in a linear array, as in Fig 3, the elements of $\mathbf{S}_{d_{m} d_{e}}$ and $\mathbf{S}_{d_{m} d_{m}}$ can be calculated to show that

$$
\mathbf{S}_{d_{m} d_{e}}=<|p|^{2}>\left[\begin{array}{llll}
\text { sinc } k r_{1} & \text { sinc } k r_{2} & \text { sinc } k r_{3} & \text { sinc } k r_{4}
\end{array}\right]
$$

where $r_{1}, r_{2}, r_{3}$ and $r_{4}$ are the distances from the monitoring microphones to the virtual microphone, and

$$
\mathbf{S}_{d_{m} d_{m}}=<|p|^{2}>\left(\begin{array}{cccc}
1 & \operatorname{sinc} k L & \operatorname{sinc} 2 k L & \operatorname{sinc} 3 k L \\
\operatorname{sinc} k L & 1 & \operatorname{sinc} k L & \operatorname{sinc} 2 k L \\
\operatorname{sinc} 2 k L & \operatorname{sinc} k L & 1 & \operatorname{sinc} k L \\
\operatorname{sinc} 3 k L & \operatorname{sinc} 2 k L & \operatorname{sinc} k L & 1
\end{array}\right)
$$

where $<|p|^{2}>$ is again the space-averaged mean squared pressure in the diffuse field and $L$ is the separation distance between the monitoring microphones. So that with regularization, 
$\mathbf{O}_{\mathrm{opt}}=\left[\begin{array}{lllll}\operatorname{sinc} k r_{1} & \operatorname{sinc} k r_{2} & \operatorname{sinc} k r_{3} & \operatorname{sinc} k r_{4}\end{array}\right]\left(\begin{array}{cccc}1+\beta & \operatorname{sinc} k L & \operatorname{sinc} 2 k L & \operatorname{sinc} 3 k L \\ \operatorname{sinc} k L & 1+\beta & \operatorname{sinc} k L & \operatorname{sinc} 2 k L \\ \operatorname{sinc} 2 k L & \operatorname{sinc} k L & 1+\beta & \operatorname{sinc} k L \\ \operatorname{sinc} 3 k L & \operatorname{sinc} 2 k L & \operatorname{sinc} k L & 1+\beta\end{array}\right)^{-1}$

In this overdetermined case it is important that the regularization factor, $\beta$ is carefully selected such that both the estimation error and condition number are acceptable. Fig. 4(a) shows the condition number and estimation error, as defined by Eq. (6), for different regularization factors when a line array of four monitoring microphones, with a separation distance along the $\mathrm{x}$-axis of $L=0.1 \mathrm{~m}$, is used to estimate the disturbance signal at a single virtual error microphone at $x=0, y=-2 L$, i.e. in front of the microphone array in Fig. 3 , in a diffuse field at a single frequency of around $135 \mathrm{~Hz}$, which corresponds to $k L=0.25$. From these results, it can be seen that the condition number is significantly reduced for values of $\beta$ above $\beta=10^{2}$ and good nearfield estimation is maintained below $\beta=10^{4}$. A good choice of the regularization factor in this case is thus $\beta=10^{3}$. It is clear from Eq. (12) that higher values of regularization will be required at low frequencies, when $k L \ll 1$, whereas when $k L \gg 1$, the matrix that is being inverted tends to the identity matrix and no regularization is required. The observation filter responses in the case considered here are shown in Fig. 4(c) as the location of the virtual error microphone is varied along the $\mathrm{x}$-axis at $y=-2 L$. For comparison, the observation filter responses with $\beta=0$ are shown in Fig. 4(b). From these results, it can be seen that with regularization, the amplitudes of the observation filter responses are significantly reduced, and also the amplitude and phase of the responses change less with the position of the virtual microphone, which indicates that the estimation will be more robust to spatial uncertainties in the position of the virtual microphone.

Fig. 5 shows contour plots of the estimation error area for different positions of the virtual error microphone on a $\pm 5 \mathrm{~L}$ grid, at different frequencies, with four monitoring microphones positioned as in Fig. 3. For these results, the regularization factors for different frequencies are determined via the process illustrated in Fig. 4 , giving $\beta=10^{3}$ at $k L=0.25, \beta=6.6 \times 10^{3}$ at $k L=0.5, \beta=6.6 \times 10^{4}$ at $k L=1$ and $\beta=0$ at $k L=2$. In these plots, the black area $(-20$ $\mathrm{dB}$ error zone) indicates the zone in which $-20 \mathrm{~dB}$ or less estimation error is achieved and 
$(a)$

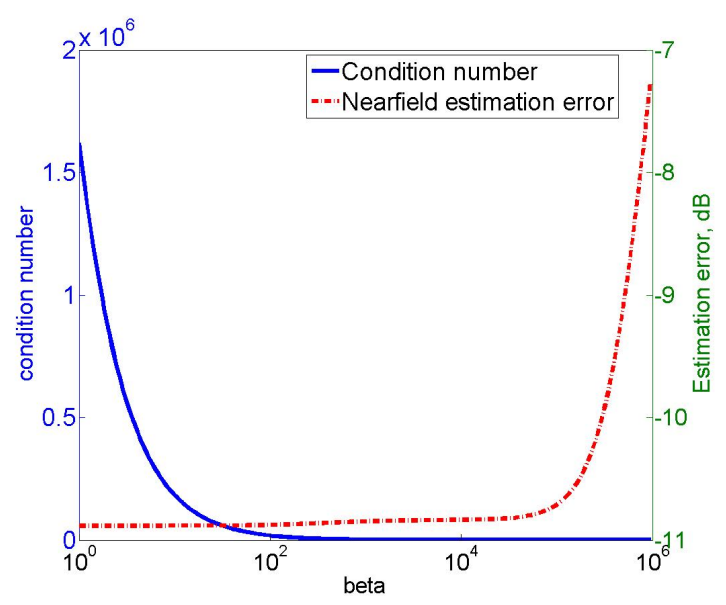

(b)
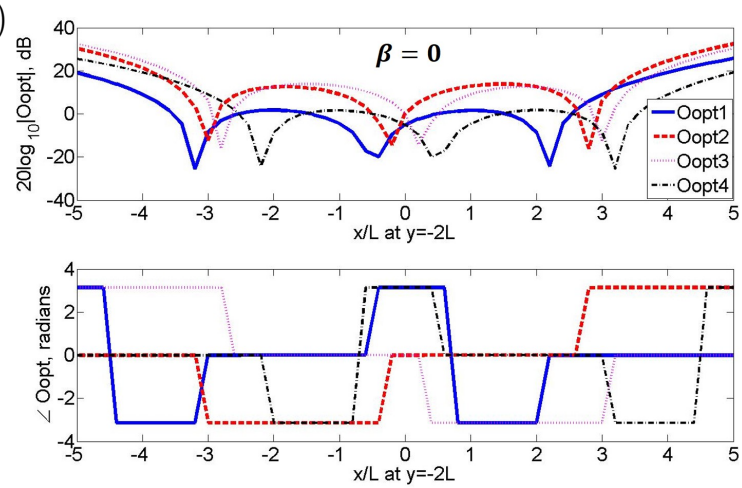

(c)
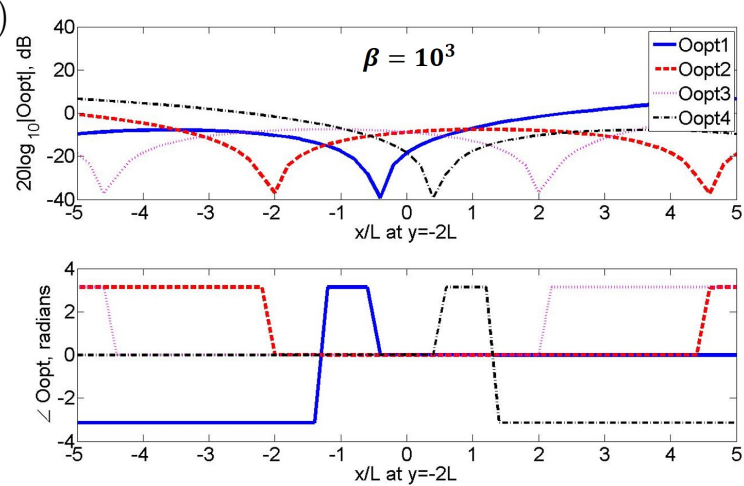

FIG. 4. (a) Condition number of the inverse term in Eq. (5) (Solid line) and the nearfield estimation error (Dash-dot line) with different regularization factors in the observation filter used to estimate disturbance signals of a virtual error microphone at $x=0, y=-2 L$ from an array of four monitoring microphones in a diffuse field. The responses of the observation filter matrix, as the location of the single virtual error microphone is varied along $\mathrm{x}$-axis, is also shown (b) without and (c) with the regularization factor $\left(\beta=10^{3}\right)$.

the gray area(-10 dB error zone) indicates the zone in which the estimation error is between $-20 \mathrm{~dB}$ and $-10 \mathrm{~dB}$. Comparing the results in Fig. 5 to the results presented for the single monitoring microphone case, it can be seen that the shapes of the zones depend on the geometry of the multiple monitoring microphones. Additionally, although the size of the zones reduces as the frequency increases, the use of the multiple monitoring microphones can clearly extend the size of the zones within which the virtual microphone signal can be accurately estimated, compared to a single monitoring microphone. For example, at $k L=0.5$, with a single monitoring microphone, the $-10 \mathrm{~dB}$ error zone is generated as a sphere of diameter $\lambda / 5.7(=2.2 L)$, from Eq. (9), but with four monitoring microphones, the length of the $-10 \mathrm{~dB}$ error zone is around $\lambda / 1.3(=9.6 \mathrm{~L})$ in the $\mathrm{x}$ direction, as shown in 
(a)

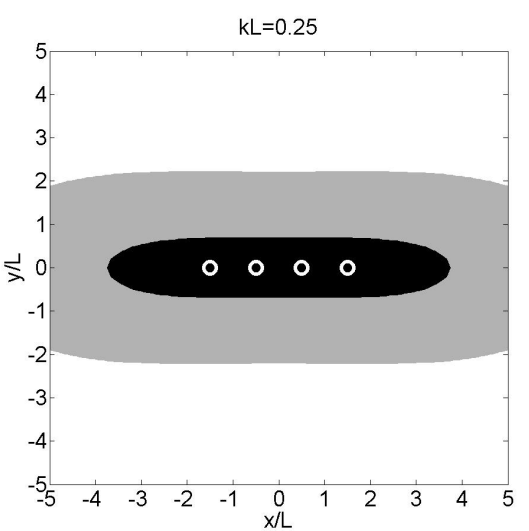

(c)

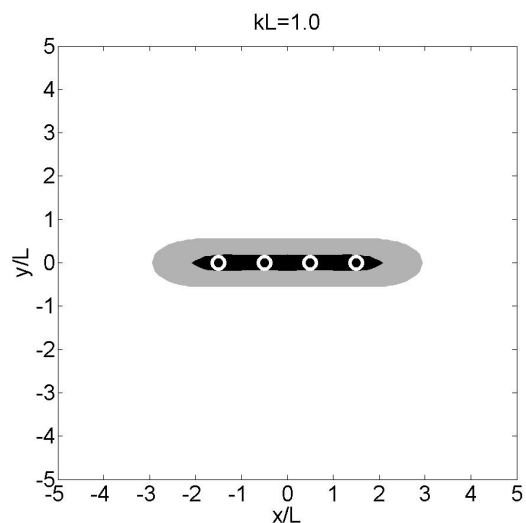

(b)

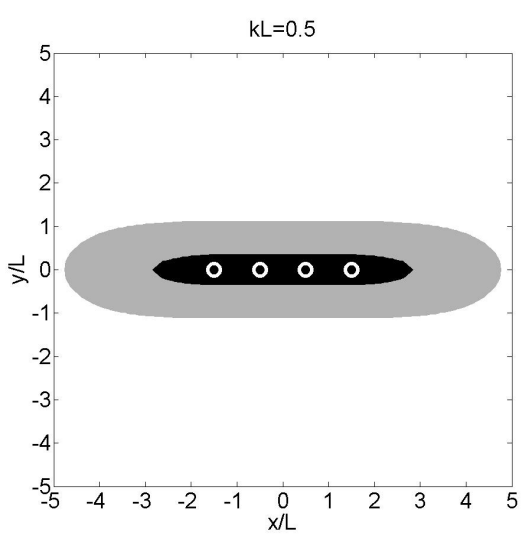

(d)

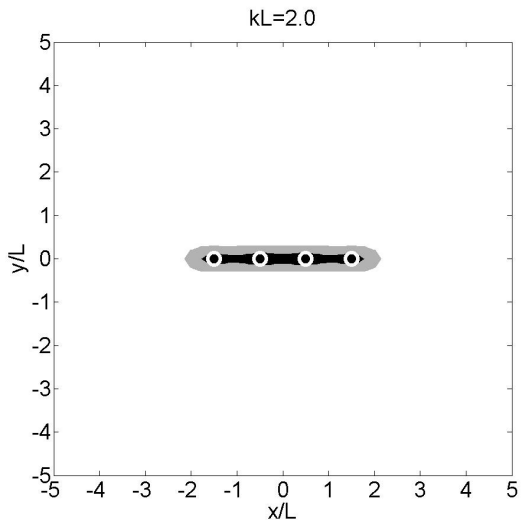

FIG. 5. The $-20 \mathrm{~dB}$ error zone(black area) in which $-20 \mathrm{~dB}$ or less estimation error is achieved and the $-10 \mathrm{~dB}$ error zone (gray area) in which an estimation error between $-20 \mathrm{~dB}$ and $-10 \mathrm{~dB}$ is achieved when four monitoring microphones at $y=0$ (white circles) are used to estimate the disturbance at different virtual error microphone locations at a single frequency in a diffuse field: (a) $k L=0.25$ with $\beta=10^{3}$, (b) $k L=0.5$ with $\beta=6.6 \times 10^{3}$, (c) $k L=1$ with $\beta=6.6 \times 10^{4}$ and (d) $k L=2$ with $\beta=0$.

Fig. 5(b).

\section{SIMULATIONS AND EXPERIMENTS IN THE FREE FIELD}

In the previous section, the performance of the remote microphone technique in an idealized diffuse field has been investigated via a number of numerical simulations. It is also interesting to investigate the influence of the spatial directivity of the primary field on the accuracy of the nearfield estimation. Therefore, this section first presents simulation results of nearfield estimation obtained with the same array of four monitoring microphones as used in the previous section, with a line array of primary sources operating at a specific location in a free field environment. Additionally, we investigate the accuracy of the nearfield 
(a)

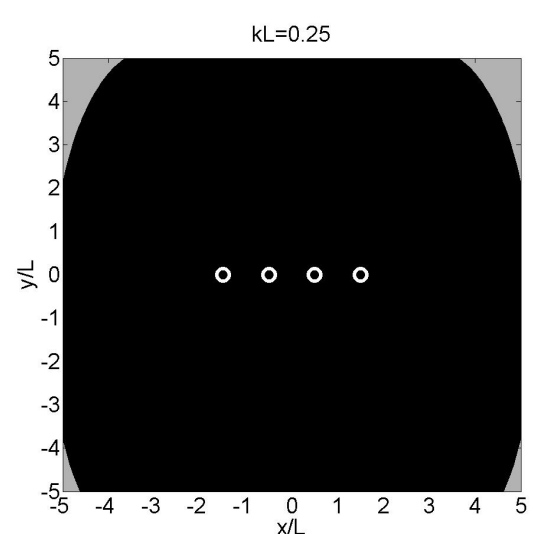

(c)

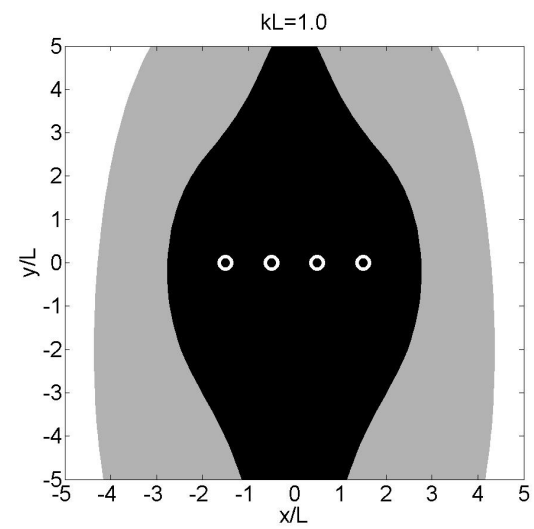

(b)

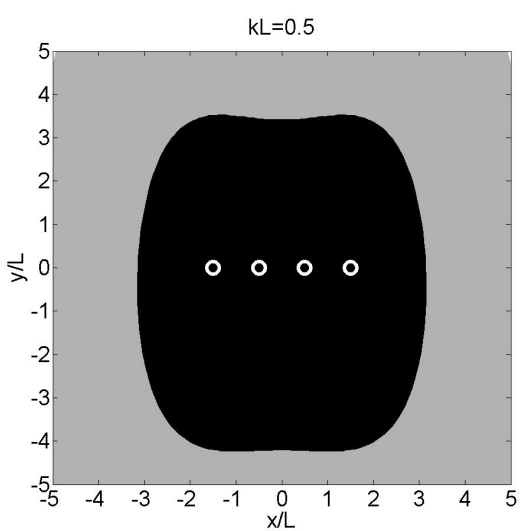

(d)

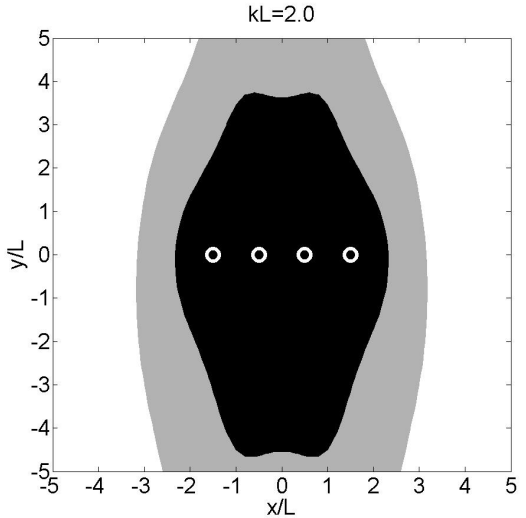

FIG. 6. The $-20 \mathrm{~dB}$ error zone(black area) in which $-20 \mathrm{~dB}$ or less estimation error is achieved and the $-10 \mathrm{~dB}$ error zone (gray area) in which an estimation error between $-20 \mathrm{~dB}$ and $-10 \mathrm{~dB}$ is achieved when four monitoring microphones at $y=0$ (white circles) are used to estimate the disturbance at different virtual error microphone locations, with 6 primary sources separated by $8 L$ at $y=30 L$, behind the microphone array in Fig. 3, producing disturbances in the free field: (a) $k L=0.25$ with $\beta=15$, (b) $k L=0.5$ with $\beta=60$, (c) $k L=1$ with $\beta=200$ and (d) $k L=2$ with $\beta=0$.

estimation that can be obtained with an experimental installation consisting of arrays of monitoring microphones around an active headrest system and a number of loudspeakers that generate disturbances in an anechoic chamber.

A. Simulation study: Estimating disturbance signals due to primary sources located on one side of the monitoring microphones

The same geometry of monitoring microphones and virtual microphones are used for the following simulations in the free field as used in Fig. 5. The locations of the primary sources, however, are different. Fig. 6 shows contour plots of the estimation error at different 
virtual error microphone locations when 6 primary sources with a inter-source spacing of $8 L$ are located at $y=30 L$, i.e. behind the microphone array in Fig. 3 if this is a plan view, and driven by uncorrelated signals. If only a single primary source is assumed for these simulations, the spatial correlation is unity at all points in the sound field and so perfect estimation can, in principle, be achieved. The regularization factors for different frequencies are again obtained through consideration of a trade-off between the nearfield estimation and the robustness to uncertainties, and the regularization factors used are: $\beta=15$ at $k L=0.25, \beta=60$ at $k L=0.5, \beta=200$ at $k L=1, \beta=0$ at $k L=2$. From the results shown in Fig. 6 , it can be seen that both the $-20 \mathrm{~dB}$ error zone and $-10 \mathrm{~dB}$ error zone in the free field are significantly larger than those in the diffuse field, since the spatial correlation between the monitoring microphones and the virtual error microphone in the free field is improved. Even at the highest frequency of about 1,082 Hz which corresponds to $k L=2$, the $-10 \mathrm{~dB}$ error zone extends to more than $10 \mathrm{~L}$ in the y-direction and this result suggests that when fixed monitoring microphones are installed on a headrest of a seat, for example, the pressure at the ears of a listener, which may be up to around $0.5 \mathrm{~m}$ away from the monitoring microphones, could be accurately estimated for primary fields propagating from one direction.

Fig. 7 shows the results of the nearfield estimation using the same monitoring microphones, but when the line array of primary sources is located at $x=-30 L$, i.e. on the right of the microphone array in Fig. 3 if this is a plan view. From these results, it can be seen that the $-20 \mathrm{~dB}$ error zone and the $-10 \mathrm{~dB}$ error zone in the $\mathrm{y}$ direction in this case are smaller than those in Fig. 6 since the relative geometry between the primary sources and the monitoring microphones in Fig. 6 maintains a higher spatial correlation between the virtual error microphone and monitoring microphones over a broader area, compared to the results in Fig. 7. It is interesting that the two error zones in the diffuse field in Fig. 5 can be approximately generated by the intersection of the corresponding zones in Fig. 6 and Fig. 7, even though the diffuse field consists of an infinite number of plane waves emanating from all possible propagation directions.

For comparison, the error zones achieved with a single monitoring microphone with the 6 primary sources to the right of the monitoring microphone are shown in Fig. 8. The spatial extent of the zones in the y direction is similar to those of the four monitoring microphone array shown in Fig. 7, but the size of the zones is rather less in the $\mathrm{x}$ direction, as may 
(a)

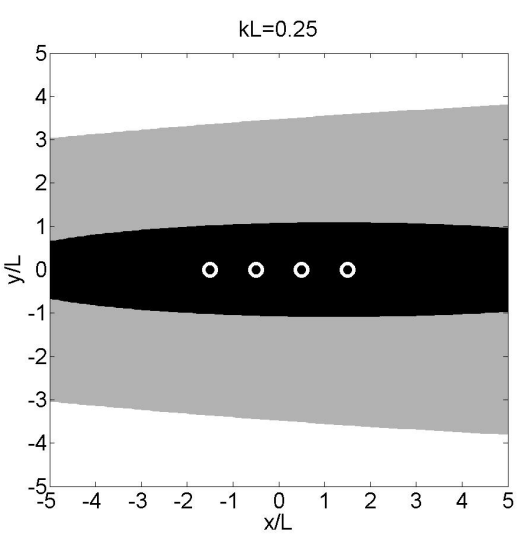

(c)

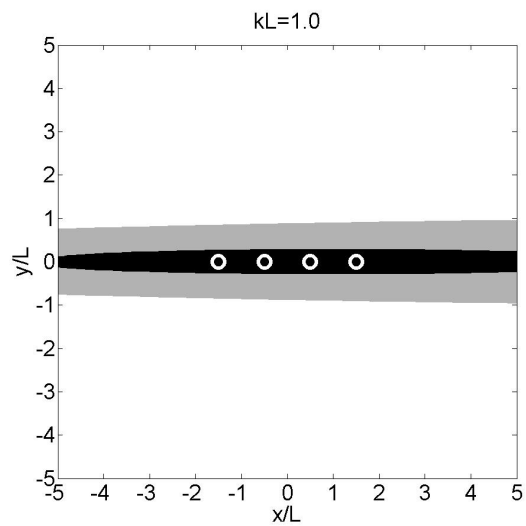

(b)

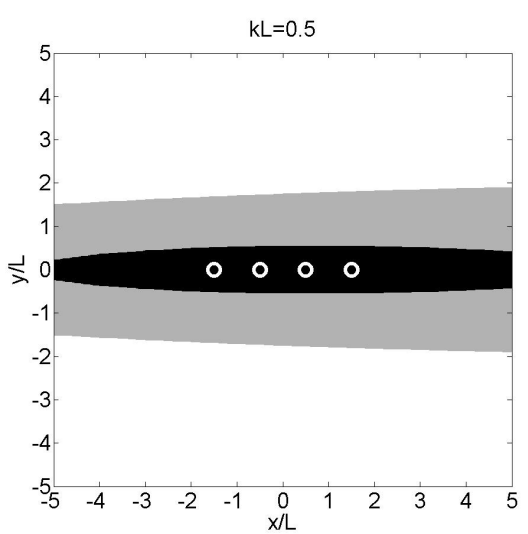

(d)

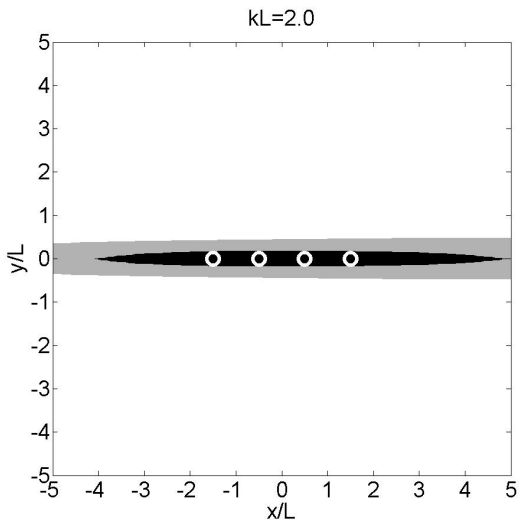

FIG. 7. The $-20 \mathrm{~dB}$ error zone(black area) in which $-20 \mathrm{~dB}$ or less estimation error is achieved and the $-10 \mathrm{~dB}$ error zone (gray area) in which an estimation error between $-20 \mathrm{~dB}$ and $-10 \mathrm{~dB}$ is achieved when four monitoring microphones at $y=0$ (white circles) are used to estimate the disturbance at different virtual error microphone locations with 6 primary sources separated by $8 L$ at $x=-30 L$, on the right of the microphone array in Fig. 3, producing disturbances in the free field: (a) $k L=0.25$ with $\beta=15$, (b) $k L=0.5$ with $\beta=60$, (c) $k L=1$ with $\beta=240$ and (d) $k L=2$ with $\beta=955$.

be expected. Note that very similar results to those in Fig. 8 are obtained with a single monitoring microphone if the 6 primary sources are behind the monitoring microphone, except that the plots are rotated by interchanging the $\mathrm{x}$ and $\mathrm{y}$ axes.

An alternative way of looking at these results is to plot the normalized estimation error at a particular point as a function of frequency, as in Fig. 9. The virtual microphone in this case is located at position $x=-L, y=-2 L$ with $L=0.1 \mathrm{~m}$, which corresponds approximately to the location of the right ear of the dummy head used in the following experiments. The results in Fig. 9 are shown for the numerical simulations with the array of four monitoring microphones in a diffuse field and with 6 uncorrelated primary sources located to the rear of the monitoring microphone array. Results when using a single monitoring microphone at 
(a)

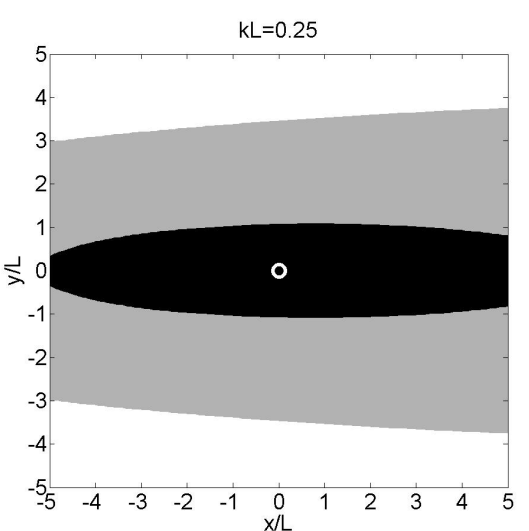

(c)

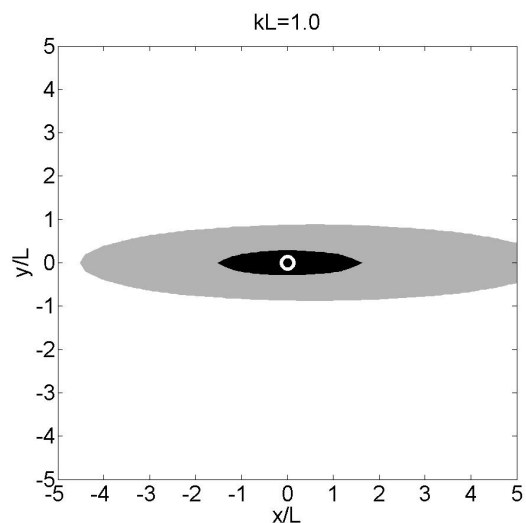

(b)

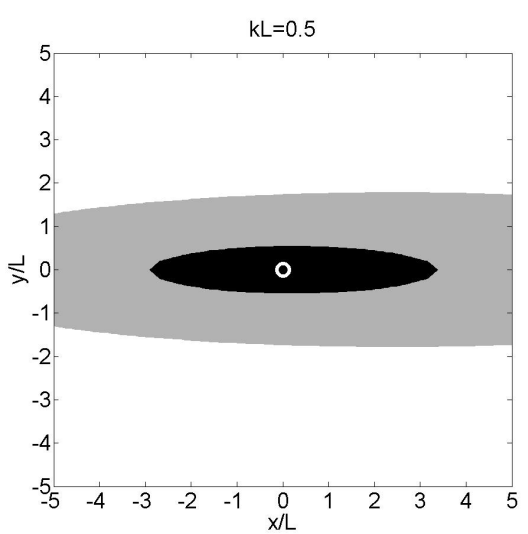

(d)

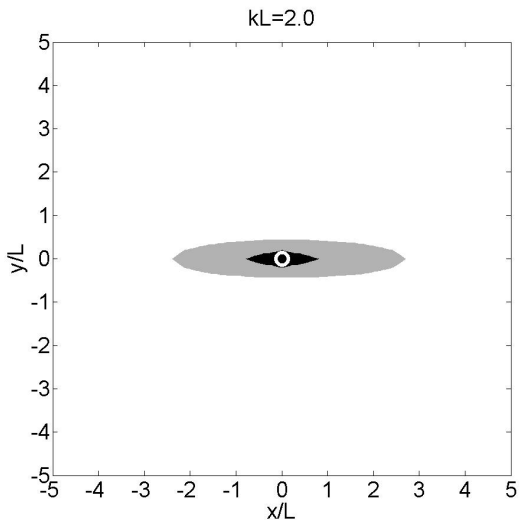

FIG. 8. The $-20 \mathrm{~dB}$ error zone(black area) in which $-20 \mathrm{~dB}$ or less estimation error is achieved and the $-10 \mathrm{~dB}$ error zone (gray area) in which an estimation error between $-20 \mathrm{~dB}$ and $-10 \mathrm{~dB}$ is achieved when a single monitoring microphones at $x=0, y=0$ (white circle) is used to estimate the disturbance at different virtual error microphone locations with 6 primary sources separated by $8 L$ at $x=-30 L$, on the right of the single microphone, producing disturbances in the free field: (a) $k L=0.25$, (b) $k L=0.5$, (c) $k L=1$ and (d) $k L=2$.

the origin to estimate the disturbance at the virtual error microphone are also shown. From these results it can be seen that in general, the estimation error increases with frequency, as expected. Although the results with the four monitoring microphone array are only slightly better than with the single monitoring microphone in the diffuse field, with the normalized error being below $-10 \mathrm{~dB}$ up to around $200 \mathrm{~Hz}$ in both cases, the estimation error with the monitoring microphone array is much less if the primary sources are behind of the array, in which case the error is below $-20 \mathrm{~dB}$ up to $1 \mathrm{kHz}$. In the latter case, the incident wave from the primary sources located to the rear is accurately detected by the monitoring microphone array before it reaches the virtual microphone position, so that its waveform can be accurately estimated. The results for the primary sources on the right of the monitoring microphones are similar to those for the diffuse field in this case and so are 


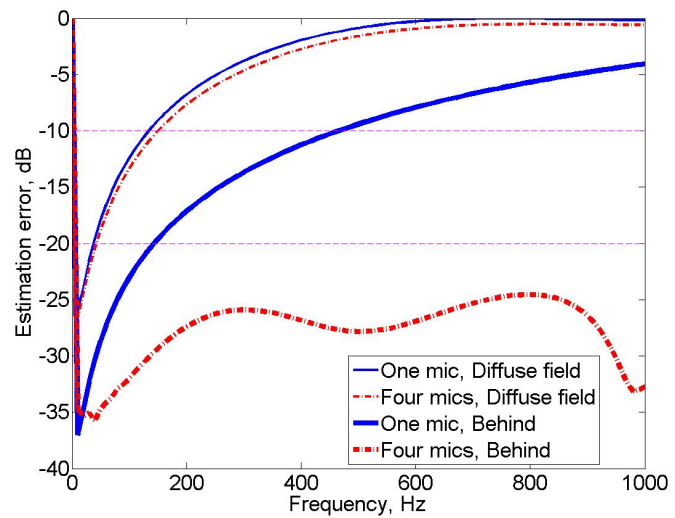

FIG. 9. The estimation error as a function of frequency when disturbance signals at a single virtual error microphone located at $x=-L, y=-2 L$ with $L=0.1 \mathrm{~m}$, which is an approximate location of the right ear of the dummy head in the following experiment, are estimated using: a single monitoring microphone(thin solid line) and four monitoring microphones(thin dashed dot line) in the diffuse field, and a single monitoring microphone(bold solid line) and four monitoring microphones(bold dashed dot line) in the free field with an array of six primary sources behind the monitoring microphone array.

not shown.

\section{B. Experimental study: Estimating disturbance signals due to primary sources in} an anechoic chamber using arrays of monitoring microphones

To estimate the effect of using the remote microphone technique in practical acoustic fields, six loudspeakers, which function as primary sources, were installed behind an active headrest system with a dummy head in an anechoic chamber, together with 24 monitoring microphones, as shown in Fig. 10. Fig. 10(b) and (c) show how these 24 monitoring microphones, located at different positions on a mounting structure and the headrest, were numbered. The aim of the experimental study was to select four monitoring microphones for the nearfield estimation, in order to investigate the relationship between the geometry of the monitoring microphone array and the accuracy of the nearfield estimation. The monitoring microphones were originally installed at many possible locations around the head. To compare the estimated disturbance signals at the virtual error microphone with the actual disturbance signals, two microphones in the ears of the dummy head were used as the physical error sensors, in order to identify the observation filter. To be consistent with the 


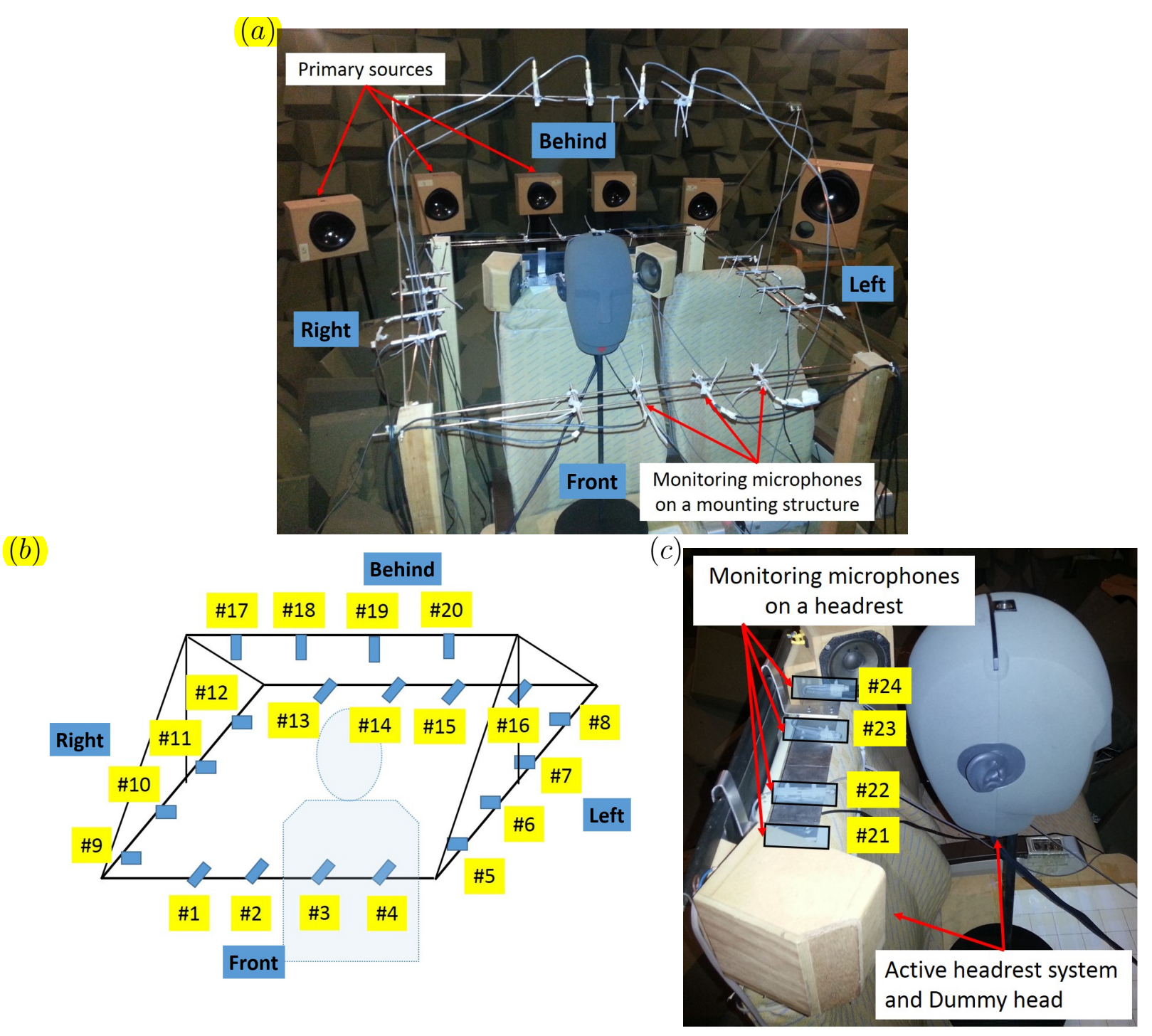

FIG. 10. (a) The overall installation for the nearfield estimation experiments when six primary sources produce uncorrelated random disturbance signals and with monitoring microphones installed around an active headrest system to estimate the disturbance signals at two error microphones in the ears of a dummy head in an anechoic chamber. (b) 20 potential monitoring microphones on a mounting structure. (c) four monitoring microphones on the headrest and two error microphones in the dummy head.

above simulations, the six loudspeakers used as the primary sources were driven with uncorrelated white noise signals. Measurements of the various acoustic transfer responses were conducted and used to calculate optimum observation filters for the various combinations of monitoring microphones, using Eq. (5), and hence the potential accuracy of the remote microphone technique has been estimated. Regularization factors for different combinations of monitoring microphones were also obtained via consideration of the trade-off between the 
estimation accuracy and the robustness, as in the previous sections.

Fig. 11(a) shows, for example, the estimation error at the ears of the dummy head, as a function of frequency, when using monitoring microphones \#21, \#22, \#23 and \#24, which are located on the headrest. This result may be compared with the simulation results in Fig. 9. The difference between the experimental results and the simulation results in the free field is mainly due to reflections from the seat, the headrest system and the dummy head. A -10 dB estimation error can be considered to be the limit for a sufficiently accurate practical estimation, because the active headrest system can then achieve $10 \mathrm{~dB}$ attenuation with this estimation error if $\mathbf{G}_{e}$ and $\mathbf{G}_{m}$ in Fig. 1 are almost identical to $\hat{\mathbf{G}}_{e}$ and $\hat{\mathbf{G}}_{m}$ [22]. Therefore, the experimental results in Fig. 11(a) show that this monitoring microphone array can accurately estimate the disturbance up to about $250 \mathrm{~Hz}$.

To investigate the effect of different selections of monitoring microphone positions on the accuracy of the nearfield estimation, the estimation error for arrays with other sets of four monitoring microphones was investigated. When monitoring microphones \#3, \#7, \#10, \#14 were selected, which form a ring around the headrest, the results are shown in Fig. 11(b). It can be seen that the estimation error with these monitoring microphones, at frequencies below $700 \mathrm{~Hz}$, is better than those in Fig. 11(a) but the estimation error in the frequency range above $700 \mathrm{~Hz}$ is degraded compared to the result in Fig. 11(a). It is found through an exhaustive search of all sets of four monitoring microphones, however, that with monitoring microphones \#13, \#22, \#23, \#16, with two microphones on the headrest and two on the rear supporting structure, the best nearfield estimation is achieved, as shown in Fig. 11(c). It is clear from the results in Fig. 11 that selection of the monitoring microphone positions should be considered with regard to both the spatial correlation of the primary field and the condition number of the inverted term in Eq. (5). For instance, in the case shown in Fig. 11(a), although the monitoring microphones are located to match with the spatial characteristics of the primary acoustic field, the small distance between the monitoring microphones results in a large condition number corresponding to the inverted term with $\beta=0$, which suggests that the observation filter will be strongly influenced by the physical and numerical uncertainties in the inverted term. To improve the robustness of this microphone array, a larger regularization factor is required up to $700 \mathrm{~Hz}$, which degrades the nearfield estimation error in this frequency range. Conversely, in Fig. 11(b), although the condition number is lower due to the larger spacing between the microphones, the formation 
(a)

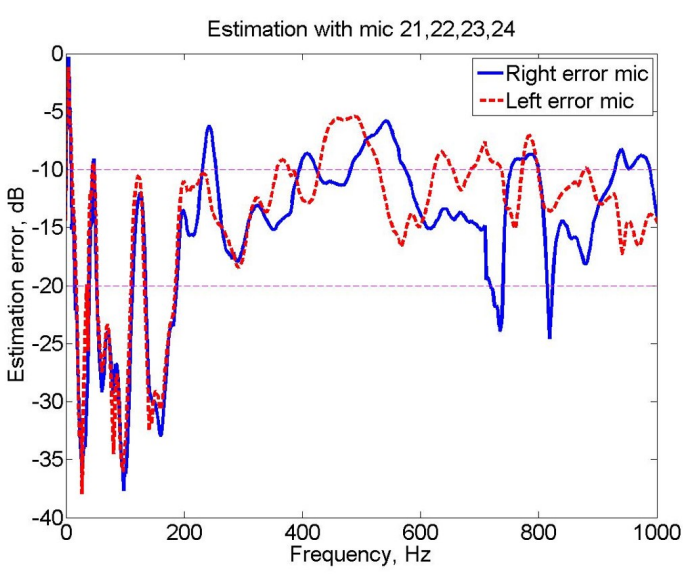

(b)

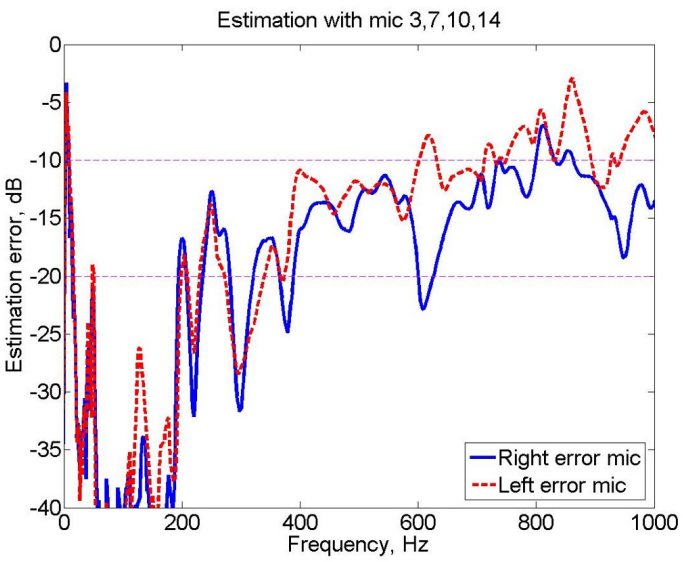

$(c)$

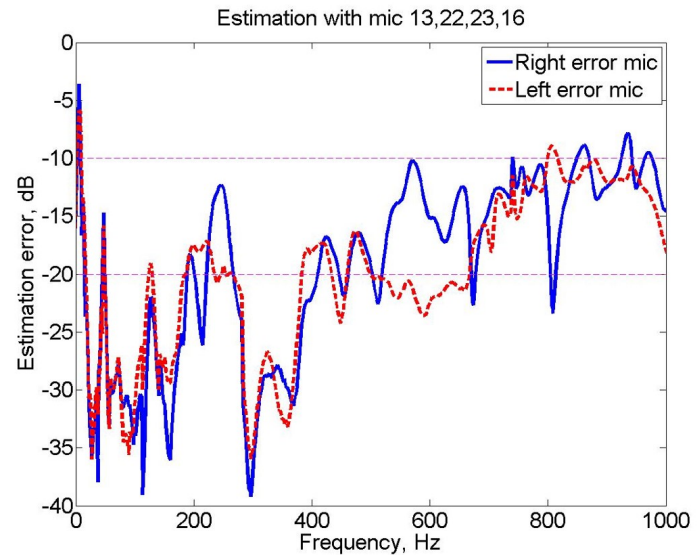

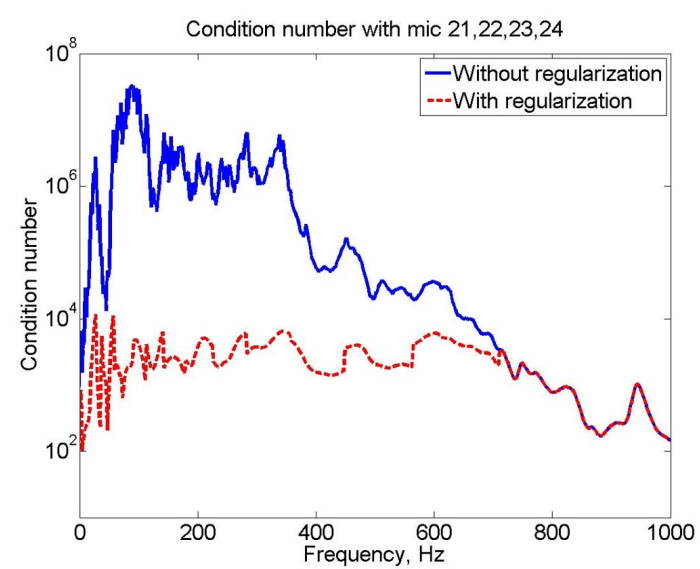
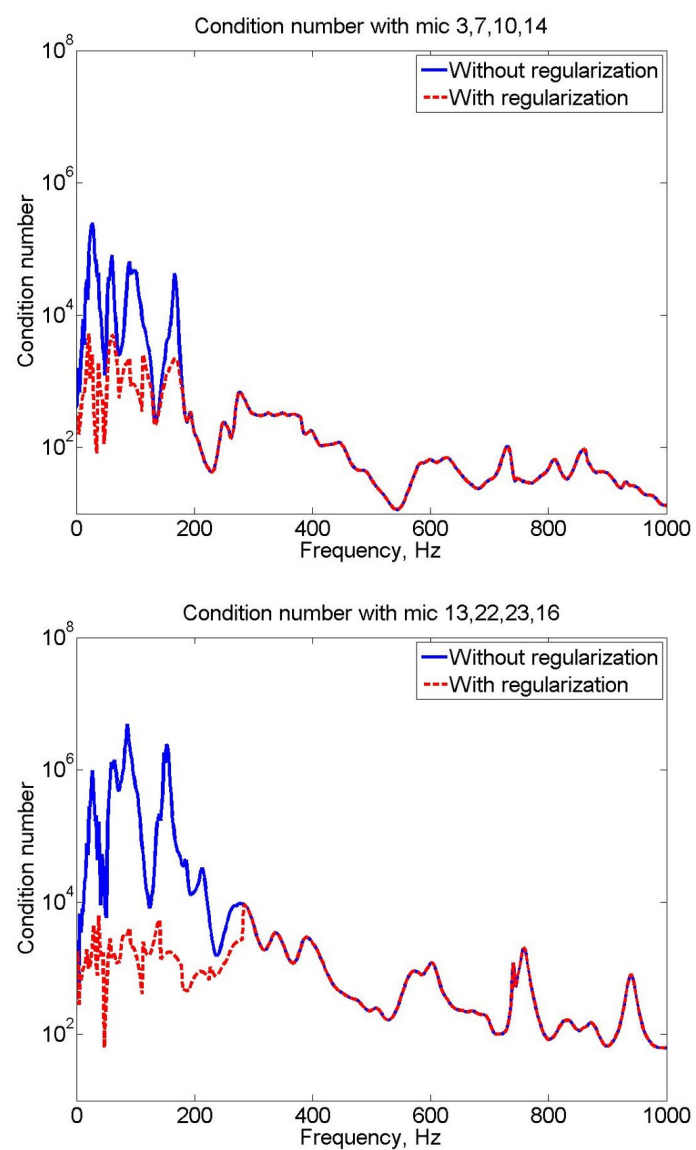

FIG. 11. The nearfield estimation error (left) and condition number (right) calculated from the measured data when different arrays of four monitoring microphones are selected from the monitoring array in Fig. 10 to estimate the disturbance signals at the ears of the dummy head when 6 primary sources located at the rear of the headrest are driven with uncorrelated white noise: (a) monitoring microphones \#21, \#22, \#23, \#24, (b) monitoring microphones \#3, \#7, \#10, \#14 and (c) monitoring microphones \#13, \#22, \#23, \#16. 
of the microphones is not well-matched with the spatial distribution of the sound field and therefore the nearfield estimation accuracy in the frequency range above $700 \mathrm{~Hz}$ is not improved. In conclusion, the monitoring microphone array in Fig. 11(c) achieves a good trade-off between spatial matching of the acoustic field and the condition number associated with the inversion. Slight asymmetries in the geometric arrays give rise to differences in the nearfield estimation error at the both ears of the dummy in Fig. 11. If the primary sources are located at different locations, different combinations of monitoring microphones need to be considered to achieve the best trade-off between the spatial properties of the primary acoustic field and the condition number.

\section{CONCLUSION}

This paper has investigated the potential for using the remote microphone technique in an active headrest system, to avoid the installation of physical error sensors at the desired cancellation positions such as the ears of a listener. A general least-squares formulation has been presented for the optimal observation filter in the frequency domain, using power and cross spectral densities of random and stationary disturbances. In this formulation, a regularization factor has been included to improve the conditioning of the matrix that needs to be inverted, which is chosen using the trade-off between the accuracy of the nearfield estimation and the conditioning of the inversion. This formulation has been used to assess the accuracy of the nearfield estimation using both numerial and experimental investigation. Although a time domain formulation for the least-squares observation filter has been presented [11], in which the constraint of causality can be imposed, in practice it is possible to use a delayed virtual error signal in an adaptive controller [21] and so the lack of causality in the frequency domain approach is not necessarily a limitation, in practice.

Simulations using this optimal observation filter have been implemented in a diffuse field. When a single monitoring microphone was used for the nearfield estimation, the observation filter is equal to the sinc $k \Delta x$ function, where $k$ is the wavenumber and $\Delta x$ is the distance from the monitoring microphone to the virtual error microphone. For simulations, an approximation to a diffuse field was synthesized using 642 uncorrelated monopole sources uniformly distributed over a sphere, and there was good agreement between the analytic results and those from simulations. An array of four monitoring microphones was also 
investigated to estimate the pressure at a single virtual error microphone in a diffuse field and the estimation regions were calculated within which a virtual microphone would have a mean-squared error of $-10 \mathrm{~dB}$ or $-20 \mathrm{~dB}$. The shapes of these zones depend on the geometry of the monitoring microphone array and the primary source locations, although the area of the zones is generally reduced as the frequency increases, as expected.

Finally, the performance of the remote microphone technique on an active headrest has been investigated via experiments in an anechoic chamber, using multiple uncorrelated primary sources to investigate the error at virtual microphones located at the ears of a dummy head. 24 monitoring microphones were installed on a mounting structure and on the headrest, and the dummy head was located in the seat. Using measured acoustical transfer responses, the results show that the choice of the monitoring microphone positions should consider both the spatial correlation of the primary field and the condition number of the inverted term of the observation filter. This paper has thus investigated the performance of the remote microphone technique under ideal and practical conditions, using both numerical models and measurements in practical arrangements. The results provide an insight into the use of the remote microphone technique in several potential applications, particularly in an active headrest system.

\section{ACKNOWLEDGMENTS}

This research is jointly funded by an EPSRC industrial CASE studentship (Award no. 14220108) with Jaguar Land Rover (JLR). The authors are especially thankful to Dr Delphine Nourzad and Mr Xavier Vinamata of JLR for their support as industrial supervisors.

[1] H. F. Olson and E. G. May, "Electronic sound absorber", J. Acoust. Soc. Am. 25(6), 1130-1136 (1953).

[2] P. A. Nelson and S. J. Elliott, Active Control of Sound, (Academic Press, London, 1992), Chap. 9\&10, pp. 310-378.

[3] M. Pawelczyk, "Adaptive noise control algorithms for active headrest system", Control Eng. Pract. 12(9), 1101-1112 (2004). 
[4] D. P. Das, D. J. Moreau and B. Cazzolato, "Performance evaluation of an active headrest using the remote microphone technique", in Proc. Acoustics 2011, Gold Coast, 69-76 (2011).

[5] P. Joseph, S. J. Elliott and P. A. Nelson, "Near field zones of quiet", J. Sound Vib. 172(5), 605-627 (1994).

[6] S. J. Elliott, P. Joseph, A. J. Bullmore and P. A. Nelson, "Active cancellation at a point in a pure tone diffuse sound field", J. Sound Vib. 120(1), 183-189 (1988).

[7] S. J. Elliott, A. David, "A virtual microphone arrangement for local active sound control", in Proc. the 1st International Conference on Motion and Vibration Control, Yokohama, 1027-1031 (1992).

[8] J. Garcia-Bonito, S. J. Elliott and C. C. Boucher, "Generation of zones of quiet using a virtual microphone arrangement", J. Acoust. Soc. Am. 101(6), 3498-3516 (1997).

[9] A. Roure and A. Albarrazin, "The remote microphone technique for active noise control", in Proc. INTER-NOISE and NOISE-CON Congress and Conference, 5, 1233-1244 (1999).

[10] D. Moreau, B. Cazzolato, A. Zander and C. Petersen, "A review of virtual sensing algorithms for active noise control", Algorithms 1(2), 69-99 (2008).

[11] S. J. Elliott and J. Cheer, "Modelling local active sound control with remote sensors in spatially random pressure fields", J. Acoust. Soc. Am. 137(4), 1936-1946 (2015).

[12] M. Adnadjevic, B. Goossens and D. Boltteldooren, "On the array configuration and accuracy of remote in-ear level sensing for in-vehicle noise control applications", Appl. Acoust. 129, 229-238 (2018).

[13] B. S. Cazzolato, "Sensing systems for active control of sound transmission into cavities", PhD thesis, The University of Adelaide, Australia (2001).

[14] C. D. Kestell, B. S. Cazzolato and C. H. Hansen, "Active noise control in a free field with virtual sensors", J. Acoust. Soc. Am. 109(1), 232-243 (1999).

[15] C. D. Petersen, R. Fraanje, B. S. Cazzolato, A. C. Zander and C. H. Hansen, "A Kalman filter approach to virtual sensing for active noise control.", MSSP 22(2), 490-508 (2008).

[16] D. Halim, L. Cheng and Z. Su, "Virtual sensors for active noise control in acousticstructural coupled enclosures using structural sensing: Robust virtual sensor design.", J. Acoust. Soc. Am. 129(3), 1390-1399 (2011).

[17] W. Jung, S. J. Elliott and J. Cheer, "Local active sound control using the remote microphone technique and head-tracking for tonal and broadband noise sources", in Proc. ICSV24, London, 
(2017).

[18] S. J. Elliott, Signal Processing for Active Control, (Academic Press, London, 2000), Chap.1, pp. 33-48.

[19] S. J. Elliott, J. Cheer, J. W. Choi and Y. Kim, "Robustness and regularization of personal audio systems", IEEE Trans. Audio, Speech, Language Process. 20(7). 2123-2133 (2012).

[20] D. J. Moreau, J. Ghan, B. S. Cazzolato and A. C. Zander, "Active noise control in a pure tone diffuse sound field using virtual sensing.", J. Acoust. Soc. Am. 125(6), 3742-3755 (2009).

[21] D. Treyer, S. Gaulocher, S. Germann and E. Curiger, "Towards the implementation of the noise-cancelling office chair: Algorithms and practical aspects", in Proc. ICSV23, Athens, (2016).

[22] W. Jung, S. J. Elliott and J. Cheer, "The effect of remote microphone technique and headtracking on local active sound control", in Proc. ICSV23, Athens, (2016). 